\title{
Hadronic contributions to the muon anomaly in the Constituent Chiral Quark Model
}

\author{
David Greynat ${ }^{1, a}$ \\ ${ }^{1}$ Dip. di Scienze Fisiche, Universitá di Napoli "Federico II", Via Cintia, 80126 Napoli, Italia \\ INFN-Sezione di Napoli, Via Cintia, 80126 Napoli, Italia
}

\begin{abstract}
The hadronic contributions to the anomalous magnetic moment of the muon which are relevant for the confrontation between theory and experiment at the present level of accuracy, are evaluated within the same framework: the constituent chiral quark model. This includes the contributions from the dominant hadronic vacuum polarization as well as from the next-to-leading order hadronic vacuum polarization, the contributions from the hadronic light-by-light scattering, and the contributions from the electroweak hadronic $Z \gamma \gamma$ vertex. They are all evaluated as a function of only one free parameter: the constituent quark mass. We also comment on the comparison between our results and other phenomenological evaluations.
\end{abstract}

\section{Introduction}

This proceeding is a summarized version of our article [1]. Our purpose is to establish a simple reference model to evaluate the various hadronic contributions to the anomalous magnetic moment of the muon $a_{\mu}$ within the same framework, and use it as a yardstick to compare with the more detailed evaluations in the literature. Indeed, the experimental world average of the anomalous magnetic moment of the muon $a_{\mu}$, assuming CPT-invariance, viz. $a_{\mu^{+}}=a_{\mu^{-}}$, is (results reported in ref. [2] and references therein)

$$
a_{\mu}^{(\exp )}=116592080(63) \times 10^{-11} \quad(0.54 \mathrm{ppm}),
$$

The prediction of the Standard Model, as a result of contributions from many physicists is (see e.g. refs. [3, 4] and references therein)

$$
a_{\mu}^{(\mathrm{SM})}=116591801(49) \times 10^{-11},
$$

where the error here is dominated at present by the lowest order hadronic vacuum polarization contribution uncertainty [5] $\left( \pm 42.0 \times 10^{-11}\right)$, as well as by the contribution from the hadronic light-by-light scattering, which is theoretically estimated to be $(105 \pm 26) \times 10^{-11}$ [6]. The results quoted in (1) and (2) imply a significant 3.6 standard deviation between theory and experiment which deserves attention. It is therefore important to reexamine critically the various theoretical contributions to Eq. (2), in particular the hadronic contributions. Ideally, one would like to do that within the framework of Quantum Chromodynamics(QCD). Unfortunatley, this demands mastering QCD at all scales from short to long distances, something which is

\footnotetext{
ae-mail: david.greynat@gmail.com
}

not under full analytic control at present. Therefore, one has to resort to experimental information whenever possible, to QCD inspired hadronic models. As a result, all the theoretical evaluations of the hadronic contributions to $a_{\mu}$ have systematic errors which are not easy to pin down rigorously.

The reference model which we propose is based on the Constituent Chiral Quark Model (C $\chi$ QM) [9]. This model emerged as an attempt to reconcile the successes of phenomenological quark models, like the De Rújula-GeorgiGlashow model [10], with QCD. The corresponding Lagrangian proposed by Manohar and Georgi (MG) is an effective field theory which incorporates the interactions of the low-lying pseudoscalar particles of the hadronic spectrum, the Nambu-Goldstone modes of the spontaneously broken chiral symmetry $\left(\mathrm{S}_{\chi} \mathrm{SB}\right)$, to lowest order in the chiral expansion [11] and in the presence of chirally rotated quark fields. Because of the $\mathrm{S}_{\chi} \mathrm{SB}$, these quark fields appear to be massive.

This model, in the presence of $S U(3)_{L} \times S U(3)_{R}$ external sources has been reconsidered by [12]. As emphasized by Weinberg [13], the corresponding effective Lagrangian is renormalizable in the Large- $\mathrm{N}_{\mathrm{c}}$ limit; however, the number of the required counterterms depends crucially on the value of the coupling constant $g_{A}$ in the model and, as shown in [12], it is minimized for $g_{A}=1$. With this choice, and a value for the constituent quark mass fixed phenomenologically, the model reproduces rather well the values of several well known low energy constants.

As discussed in ref. [12] the $\mathrm{C} \chi \mathrm{QM}$ model has, however, its own limitations. Applications to the evaluation of low-energy observables involving the integration of Green's functions over a full range of euclidean momenta fail, in general, because there is no matching of the model 
to the QCD short-distance behaviour. There is, however, an exceptional class of low-energy observables for which the MG-Lagrangian predictions can be rather reliable [12].

We present in this proceeding a summary of the different evaluations with the $\mathrm{C} \chi \mathrm{QM}$ Lagrangian which has the advantage of simplicity and can provide a consistency check with the more elaborated phenomenological approaches.

\section{Hadronic Vacuum Polarization}

Since the dominant contribution to the muon anomaly from the hadronic vacuum polarization shown in Fig. (1) is a well known representation [14] we use it to determine the only free parameter in the $\mathrm{C} \chi \mathrm{QM}$ Lagrangian the constituent quark mass $M_{Q}{ }^{1}$.

This contribution

$$
a_{\mu}^{(\mathrm{HVP})}=\int_{4 M_{Q}^{2}}^{\infty} \frac{d t}{t} K\left(\frac{t}{m_{\mu}^{2}}\right) \frac{1}{\pi} \operatorname{Im} \Pi^{(\mathrm{HVP})}(t),
$$

where $^{2}$

$$
K\left(\frac{t}{m_{\mu}^{2}}\right)=\left(\frac{\alpha}{\pi}\right) \int_{0}^{1} d x \frac{x^{2}(1-x)}{x^{2}+\frac{t}{m_{\mu}^{2}}(1-x)},
$$

and $\frac{1}{\pi} \operatorname{Im} \Pi(t)$ denotes the electromagnetic hadronic spectral function.

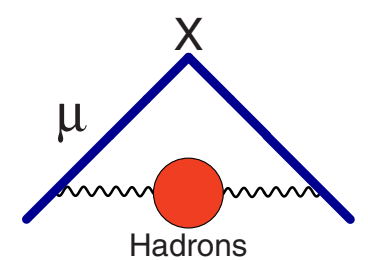

Figure 1. Hadronic Vacuum Polarization contribution to the Muon Anomaly.

The constituent quark fields in the $\mathrm{C} \chi \mathrm{QM}$ are assumed to have gluonic interactions, since the Goldstone modes are already in the Lagrangian, the color-SU(3) coupling constant is supposed to be no longer running below a scale $\mu_{0} \simeq 2 \mathrm{GeV}$ where $\alpha_{s}\left(\mu_{0}\right) \simeq 0.33$ and non-perturbative effects become significant. With inclusion of the leading gluonic corrections in perturbation theory, and to leading

${ }^{1}$ There are many estimates of this contribution, as well as some of the higher order ones, with quark models which can be found in the literature. An earlier reference is [16] and two more recent ones [17] and [18].

${ }^{2}$ The analytic expression of $K\left(t / m_{\mu}^{2}\right)$ was first given in ref. [15]; see also ref. [20]. order in Large- $\mathrm{N}_{\mathrm{c}}$, the spectral function in Eq. (3) is then

$$
\begin{aligned}
& \frac{1}{\pi} \operatorname{Im} \Pi^{(\mathrm{HVP})}(t) \\
& =\left(\frac{\alpha}{\pi}\right) N_{c} \frac{2}{3}\left\{\delta\left(\frac{1}{2}-\frac{1}{6} \delta^{2}\right)\right. \\
& \left.+\left[\frac{N_{c} \alpha_{\mathrm{S}}\left(\mu_{0}\right)}{\pi} \frac{3}{8} \theta\left(\mu_{0}^{2}-t\right)+\frac{N_{c} \alpha_{\mathrm{S}}(\sqrt{t})}{\pi} \frac{3}{8} \theta\left(t-\mu_{0}^{2}\right)\right] \rho_{\mathrm{KS}}(t)\right\},
\end{aligned}
$$

where $\rho_{\mathrm{KS}}(t)$ can be extracted from the early QED calculation of Källen and Sabry [19] (see also ref. [20]):

$$
\begin{aligned}
& \rho_{\mathrm{KS}}(t) \\
& =\delta\left(\frac{5}{8}-\frac{3}{8} \delta^{2}-\left(\frac{1}{2}-\frac{1}{6} \delta^{2}\right) \log \left[64 \frac{\delta^{4}}{\left(1-\delta^{2}\right)^{3}}\right]\right) \\
& +\left(\frac{11}{16}+\frac{11}{24} \delta^{2}-\frac{7}{48} \delta^{4}\right. \\
& \left.\quad+\left(\frac{1}{2}+\frac{1}{3} \delta^{2}-\frac{1}{6} \delta^{4}\right) \log \left[\frac{(1+\delta)^{3}}{8 \delta^{2}}\right]\right) \log \left[\frac{1+\delta}{1-\delta}\right] \\
& +2\left(\frac{1}{2}+\frac{1}{3} \delta^{2}-\frac{1}{6} \delta^{4}\right)\left(2 \operatorname{Li}_{2}\left[\frac{1-\delta}{1+\delta}\right]+\operatorname{Li}_{2}\left[-\frac{1-\delta}{1+\delta}\right]\right) .
\end{aligned}
$$

Also, at the level of the accuracy expected from the $\mathrm{C} \chi \mathrm{QM}$, it is sufficient to use the one loop expression

$$
\frac{\alpha_{\mathrm{s}}(\sqrt{t})}{\pi} \simeq \frac{1}{\left(\frac{11}{6} N_{c}-\frac{n_{f}}{3}\right) \log \frac{\sqrt{t}}{\Lambda}},
$$

with $\Lambda \simeq 250 \mathrm{MeV}$ and $n_{f}=3$. In order to compare the $\mathrm{C} \chi \mathrm{QM}$ results for $a_{\mu}^{(\mathrm{HVP})}$ with the phenomenological determinations which incorporate experimental data, we still have to estimate of the $1 / \mathrm{N}_{\mathrm{c}}$-suppressed effects, we then consider the contributions from the $\pi^{+} \pi^{-}$and $K^{+} K^{-}$ intermediate states to the spectral function in Eq. (3), as predicted by the $\mathrm{C} \chi \mathrm{QM}$. Notice that in this evaluation, the point like coupling $(-i e)\left(p^{\mu}-p^{\mu}\right)$ of scalar QED is replaced by the dressed coupling:

$$
(-i e)\left(p^{\mu}-p^{\prime \mu}\right) \Rightarrow(-i e)\left(p^{\mu}-p^{\prime \mu}\right)\left\{1+\mathcal{F}\left(Q^{2}\right)\right\},
$$

with $\mathcal{F}\left(Q^{2}\right)$ the pion (kaon) electromagnetic form factor of the $\mathrm{C} \chi \mathrm{QM}$ which, for $g_{A}=1$, is given by the expression:

$$
\begin{aligned}
\mathcal{F}\left(Q^{2}\right) & =\frac{\mathrm{N}_{\mathrm{c}}}{16 \pi^{2}} \frac{Q^{2}}{f_{\pi}^{2}}\left(-\frac{4 M_{Q}^{2}}{Q^{2}}\right) \\
& \times\left[1+\frac{1}{2} \sqrt{1+\frac{4 M_{Q}^{2}}{Q^{2}}} \log \frac{\sqrt{1+\frac{4 M_{Q}^{2}}{Q^{2}}}-1}{\sqrt{1+\frac{4 M_{Q}^{2}}{Q^{2}}}+1}\right] .
\end{aligned}
$$

The form factor $\mathcal{F}\left(Q^{2}\right)$, however, does not match the QCD behaviour at large- $Q^{2}$ values and, therefore, the estimate we propose for the $1 / \mathrm{N}_{\mathrm{c}}-$ suppresed contributions to the the muon anomaly can only be considered as reasonable 
up to values of $t$ in Eq. (3) below $t \sim \mu_{0}$ where the asymptotic pQCD regime sets in. Contributions beyond $t \sim \mu_{0}$ have already been taken into account by the second term of the spectral function in Eq. (5).

These considerations provide us with a framework to fix the constituent quark mass $M_{Q}$. The prediction of the $\mathrm{C} \chi \mathrm{QM}$, as described above, should be compared to the phenomenological contribution from hadrons formed of $u$, $d$ and $s$ quarks only, at the level of one-photon exchange. Contributions like for example the one from an intermediate $\pi^{0} \gamma$ state should therefore be excluded so far (more on that later on), as well as those involving $c, b$ and $t$ quarks. From the numbers quoted in TABLE II of ref. [5], we then find that this restriction reduces the phenomenological determination of the anomaly from hadronic vacuum polarization to a central value

$$
\left.a_{\mu}^{(\mathrm{HVP})}\right|_{\text {phen. }} \simeq 653 \times 10^{-10}
$$

shows that fixing $M_{Q}$ in the range

$$
M_{Q}=(240 \pm 10) \mathrm{MeV}
$$

reproduces the phenomenological determination within an error of less than $10 \%$. This determination of the constituent quark mass is the value which we shall systematically use for $M_{Q}$ when evaluating the predictions for the other hadronic contributions to the muon anomaly. We wish to emphasize, however, that the error of $10 \mathrm{MeV}$ in Eq. (11) only reflects the phenomenological choice that we have made in order to fix $M_{Q}$. As discussed in the Introduction the $\mathrm{C} \chi \mathrm{QM}$ is only a model of low energy QCD and, as such, there is no a priori way to fix $M_{Q}$ from first principles. The error in Eq. (11) does not reflect the systematic error due to other plausible ways of fixing $M_{Q}$.

\section{Hadronic Vacuum Polarization Contributions at Next-to-Leading Order}

After having fixed the constituent quark mass, it is then possible to evaluate next-to-leading order contributions to $a_{\mu}$. For obvious reason, we will present here only the different diagram classification that we used and the results obtained. All details and complete discussion can be found in [1].

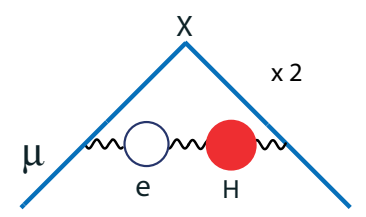

Figure 3. Feynman diagrams corresponding to the Class B contribution to the muon anomaly.

As we can see in table 1, we obtain quite reasonable results except from the contribution in the class D coming from the $\pi^{0} \gamma$ intermediate state. In order to understand this, we can simplify the phenomenological determination as much as possible to see where the big contribution

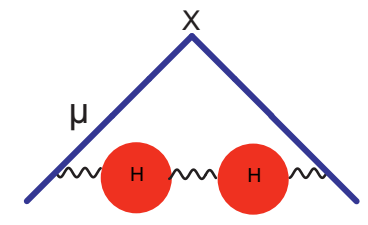

Figure 4. HVP Contributions at $O(\alpha)$ corresponding to the Class C.

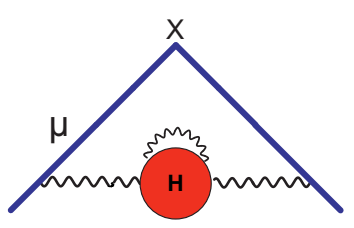

Figure 5. Feynman diagrams corresponding to the Class D contribution to the muon anomaly.

\begin{tabular}{|c|c|c|}
\hline \hline Class & Result $\times 10^{-11}$ & Pheno. $\times 10^{-11}$ \\
\hline \hline $\mathrm{A}$ & $-171 \pm 10$ & $-(207.3 \pm 1.9)[24]$ \\
$\mathrm{B}$ & $89 \pm 7$ & $106.0 \pm 0.9[24]$ \\
$\mathrm{C}$ & $2.2 \pm 0.3$ & $3.4 \pm 0.1[24]$ \\
& & $3.0 \pm 0.1[31]$ \\
$\mathrm{D}(\pi \gamma)$ & $2.2 \pm 0.1$ & $44.2 \pm 1.9[5,24]$ \\
$\mathrm{D}(\mathrm{Q}-$ loop $)$ & $13.5 \pm 0.5$ & \\
\hline Total & $-64 \pm 12$ & $-98.4 \pm 0.6_{\exp } \pm 0.4_{\mathrm{rad}}[30]$ \\
\hline \hline
\end{tabular}

Table 1. Results for the HVP contributions of $O\left(\frac{\alpha}{\pi}\right)^{3}$ in the $\mathrm{C} \chi \mathrm{QM}$.

comes from. For that, it will be sufficient to approximate this contribution as follows:

$$
a_{\mu}^{(\pi \gamma)} \sim\left(\frac{\alpha}{\pi}\right) \int_{m_{\pi}^{2}}^{\infty} \frac{d t}{t} \frac{1}{3} \frac{m_{\mu}^{2}}{t} \frac{1}{\pi} \operatorname{Im} \Pi^{(\pi \gamma)}(t),
$$

and use a narrow width expression for the spectral function, which as we shall soon see, is dominated by the $\omega$ contribution. This results in the simple formula:

$a_{\mu}^{(\pi \gamma)} \sim \frac{1}{3} \frac{m_{\mu}^{2}}{M_{\omega}^{2}} \frac{4}{\pi} \frac{\Gamma\left(\omega \rightarrow e^{+} e^{-}\right)}{M_{\omega}} \frac{\Gamma\left(\omega \rightarrow \pi^{0} \gamma\right)}{\Gamma_{\omega}}=53 \times 10^{-11}$,

which reproduces, in order of magnitude, the phenomenological estimates. We can, therefore, see that the big number comes from the large experimental value of the branching ratio

$$
\frac{\Gamma\left(\omega \rightarrow \pi^{0} \gamma\right)}{\Gamma_{\omega}} \simeq 8 \times 10^{-2}
$$

Notice that in the case of the $\rho$ contribution the corresponding branching ratio is much smaller:

$$
\frac{\Gamma\left(\rho \rightarrow \pi^{0} \gamma\right)}{\Gamma_{\rho}} \simeq 6 \times 10^{-4}
$$

It is the large branching ratio in Eq. (14) which the $\mathrm{C} \chi \mathrm{QM}$ fails to reproduce! 
Phenomenologically, the large branching ratio $\frac{\Gamma\left(\omega \rightarrow \pi^{0} \gamma\right)}{\Gamma\left(\rho \rightarrow \pi^{0} \gamma\right)}$ is due to the $\omega-\phi$ mixing and the fact that the $\phi$ is an almost pure $s \bar{s}$ state. By construction, the $\mathrm{C} \chi \mathrm{QM}$ form factor is $S U(3)$ invariant and, therefore, like any model which is $S U(3)$ invariant, fails to reproduce this phenomenological fact.

\section{Hadronic light-by-light contribution}

In the $\mathrm{C} \chi \mathrm{QM}$ there are two types of contributions: the Constituent Quark Loop (CQL) contribution shown in Fig. (6) and the Goldstone Exchange Contribution shown

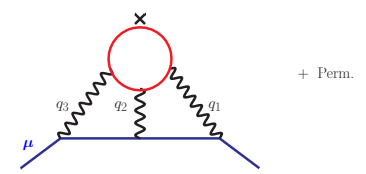

Figure 6. Constituent Quark Loop Contribution to the Muon Anomaly in the $\mathrm{C} \chi \mathrm{QM}$.

in Fig. (7) with constituent quark loops at each vertex. We shall consider these two types of contributions, both leading in the $1 / \mathrm{N}_{\mathrm{c}}$-expansion, separately.

This contribution can be obtained from the QED analytic calculation of Laporta and Remmidi [33] with the result

$$
a_{\mu}^{(\mathrm{HLbyL})}\left(\mathrm{CQL}, M_{Q}=240 \mathrm{MeV}\right)=82.2 \times 10^{-11} .
$$

The second contribution coming from the $\pi^{0}$ exchange lies on [36] the evaluation of the vertex $\pi^{0} \gamma^{*} \gamma^{*}$ form factor in the $\mathrm{C} \chi \mathrm{QM}$,

$$
\mathcal{F}_{\pi^{*} \gamma^{*} \gamma^{*}}^{(\chi \mathrm{QM})}\left(q_{2}^{2}, q_{1}^{2}, q_{3}^{2}\right)=-\frac{i e^{2} N_{c}}{12 \pi^{2} f_{\pi}} \int_{0}^{1} d x x \int_{0}^{1} d y \frac{2 M_{Q}^{2}}{\mathbb{D}},
$$

whith

$\mathbb{D}=M_{Q}^{2}-x(1-x)(1-y) q_{1}^{2}-x^{2} y(1-y) q_{2}^{2}-x y(1-x) q_{3}^{2}$

Numerically, one can evaluate this contribution and obtain,

$$
a_{\mu}^{(\mathrm{HLbyL})}\left(\pi^{0}\right)_{\chi \mathrm{QM}}=(68 \pm 3) \times 10^{-11},
$$

which does not include the systematic error of the model, agrees well with the phenomenological determinations of this contribution which, according to the most recent update [39] and depending on the underlying phenomenological model for the form factors $\mathcal{F}_{\pi^{0 *} \gamma^{*} \gamma^{*}}^{(\chi \mathrm{QM})}\left(q_{2}^{2}, q_{1}^{2}, q_{3}^{2}\right)$ vary between

$$
a_{\mu}^{(\mathrm{HLbyL})}\left(\pi^{0}\right)_{\text {phen. }}=(57.4 \pm 4.6) \times 10^{-11}
$$

and

$$
a_{\mu}^{(\mathrm{HLbyL})}\left(\pi^{0}\right)_{\text {phen. }}=(80.1 \pm 4.7) \times 10^{-11} .
$$

It is a fact that asymptotically, for $M_{Q} \rightarrow \infty$, the $\pi^{0}$ contribution largely dominates the Constituent Quark Loop contribution:

$$
\left.\frac{a_{\mu}^{(\mathrm{HLbyL})}\left(\pi^{0}\right)}{a_{\mu}^{(\mathrm{HLbyL})}(\mathrm{CQL})}\right|_{M_{Q} \rightarrow \infty} \sim \frac{N_{c} M_{Q}^{2}}{16 \pi^{2} f_{\pi}^{2}} \frac{1}{\zeta(3)-\frac{19}{24}} \log ^{2} \frac{M_{Q}}{m_{\pi}}
$$

however, this asymptotic behaviour is far from being reached at values of $M_{Q}$ between $230 \mathrm{MeV}$ and $250 \mathrm{MeV}$, for which the Constituent Quark Loop contribution still dominates over the Goldstone contribution.

For the total hadronic light-by-light contribution in the $\mathrm{C} \chi \mathrm{QM}$, which includes the quark loop contribution as well as the $\pi^{0}$-exchange contributions, we then find

$$
148 \times 10^{-11} \leq a_{\mu}^{(\mathrm{HLbyL})}(\mathrm{C} \chi \mathrm{QM}) \leq 153 \times 10^{-11}
$$

for $250 \mathrm{MeV} \geq M_{Q} \geq 230 \mathrm{MeV}$. This result, which does not include the systematic error of the model, has to be compared with the phenomenological estimate

$$
a^{(\mathrm{HLbyL})}=(122 \pm 18) \times 10^{-11},
$$

for the total of the hadronic contributions not suppressed in the $1 / \mathrm{N}_{\mathrm{c}}-$ expansion (see e.g. ref. [40] for details). Within the expected systematic uncertainties they compare rather well.

The interesting feature which emerges from this calculation is the observed balance between the Goldstone contribution and the Quark Loop contribution. Indeed, as the constituent quark mass $M_{Q}$ gets larger and larger, the Goldstone contribution dominates; while for $M_{Q}$ smaller and smaller it is the Quark Loop contribution which dominates. This is illustrated by the plot of the total $a_{\mu}^{\text {(HLbyL) }}(\mathrm{C} \chi \mathrm{QM})$ versus $M_{Q}$ shown in Fig. (8). What this plot shows is in flagrant contradiction with the results reported in ref. [41] based in a calculation using a DysonSchwinger inspired model. In this model, the authors find a contribution from the $\pi^{0}$-exchange which, within errors, is compatible with the other phenomenological determinations and, in particular, with our $\mathrm{C} \chi \mathrm{QM}$ result in Eq. (19); yet their result for the equivalent contribution to the quark loop turns out to be almost twice as large with a total contribution

$$
a^{(\mathrm{HLbyL})}\left(\text { ref. [41]) }=(217 \pm 91) \times 10^{-11} .\right.
$$

The central value of this result would require a ridicously small value of $M_{Q}$ in order to be reproduced by the $\mathrm{C} \chi \mathrm{QM}$ and, furthermore, for such a small value of $M_{Q}$ the $\pi^{0}$ exchange contribution would be far too small as compared to all the phenomenological estimates, including the one in ref. [41]. We conclude that a range of values such as

$$
170 \leq a_{\mu}^{(\mathrm{HLbyL})} \times 10^{11} \leq 308,
$$

allowed by the result quoted in Eq. (25), cannot be digested within the $\mathrm{C} \chi \mathrm{QM}$ and in our opinion this casts serious doubts about the compatibility of the model used in ref. [41] with basic QCD features. 


\section{Hadronic Electroweak Contributions}

These are the contributions to the muon anomaly which appear at the two-loop level in the electroweak sector. They are the ones generated by the hadronic $\gamma \gamma Z$ vertex, with one $\gamma$ and the $Z$-boson attached to a muon line, as illustrated by the Feynman diagrams in Fig. (9).
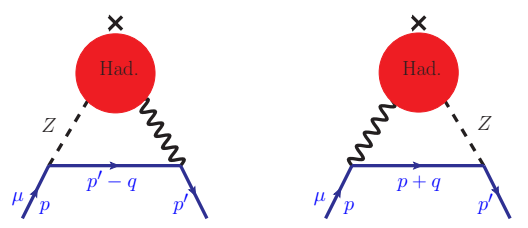

Figure 9. Feynman diagrams with the hadronic $\gamma \gamma Z$ vertex which contributes to the muon anomaly.

These contributions are particularly interesting because, a priori, they could be enhanced by a large $\log \left(M_{Z}^{2} / m_{\mu}^{2}\right)$ factor. However, due to the anomaly-free coupling assignments in the Standard Model, there is an important cancellation of UV-scales between the lepton and the quark contributions within a given family [42, 43]. What is left out of this cancellation in the sector of the $u, d$ and $s$ quarks, where the strong interactions play a subtle role at long distances, is governed by the dynamics of spontaneous chiral symmetry breaking [42, 44-47].

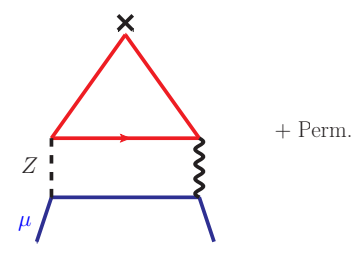

Figure 10. Feynman diagrams in the $\mathrm{C} \chi \mathrm{QM}$ of the $\gamma \gamma Z$ vertex type.

The $\mathrm{C} \chi \mathrm{QM}$, where the hadronic blob in Fig. (9) is replaced by a constituent quark loop as illustrated in Fig. (10), offers a simple way to estimate these contributions as, with $g_{A}=1$, for $m_{c}=1.5 \mathrm{GeV}$ and $M_{Q}=$ $240 \mathrm{MeV}$,

$$
\begin{aligned}
& \left.a_{\mu}^{(\mathrm{HEW})}\right|_{e, u, d ; \mu, s, c}(\mathrm{C} \chi \mathrm{QM}) \\
& =\frac{G_{\mathrm{F}}}{\sqrt{2}} \frac{m_{\mu}^{2}}{8 \pi^{2}} \frac{\alpha}{\pi}\left(-\frac{2}{3} \log \frac{M_{Q}^{2}}{m_{\mu}^{2}}-4 \log \frac{m_{c}^{2}}{m_{\mu}^{2}}-5+\frac{8}{9} \pi^{2}\right) \\
& =-5.0 \times 10^{-11}
\end{aligned}
$$

with the systematic errors of the model, is compatible with the phenomenological determination [46]:

$$
\left.a_{\mu}^{(\mathrm{HEW})}\right|_{e, u, d ; \mu, s, c}=(-6.7 \pm 0.5) \times 10^{-11} .
$$

\section{Conclusions}

From the previous considerations we conclude that the $\mathrm{C} \chi \mathrm{QM}$ provides a useful and simple reference model to evaluate the hadronic contributions to the anomalous magnetic moment of the muon. The effective Lagrangian of this model is renormalizable in the Large- $\mathrm{N}_{\mathrm{c}}$ limit [13] and, as shown in [12], the number of the required counterterms in this limit is minimized for a choice of the axial coupling: $g_{A}=1$. The only free parameter of the model is then the mass of the constituent quark mass $M_{Q}$ which in Section II, from a comparison with the phenomenological determination of the lowest order hadronic vacuum polarization contribution to the muon anomaly, has been fixed to

$$
M_{Q}=(240 \pm 10) \mathrm{MeV},
$$

This range of values for $M_{Q}$ reproduces the phenomenological determination within an error of less than $10 \%$. All the other hadronic contributions have then been evaluated for this range of values of $M_{Q}$ with the results which are summarized in Table 2, despite one exception from the contribution from the $\pi^{0} \gamma$ intermediate state to hadronic vacuum polarization where the $\mathrm{C} \chi \mathrm{QM}$, because of its $S U(3)$ invariance, fails to reproduce the phenomenological determination which is particularly enhanced because of the large observed branching ratio in Eq. (14).

\begin{tabular}{|c|c|}
\hline \hline Class & Result in $10^{-10}$ units \\
\hline \hline HVP & $652+47$ \\
& -42 \\
HVP to $O\left(\frac{\alpha}{\pi}\right)^{3}$ & $-6.4 \pm 1.2$ \\
HLbyL & $15.0 \pm 0.3$ \\
HEW & -0.5 \\
\hline \hline
\end{tabular}

Table 2. Summary of results for the hadronic contributions to the muon anomly in the $\mathrm{C} \chi \mathrm{QM}$.

\section{Acknowledgments}

The author thanks the organizers, in particular G. D'Ambrosio, for this nice Workshop. The author is also graceful to E. de Rafael for the discussions and suggestions about the presentation and proceeding. This work was supported in part by MIUR under project 2010YJ2NYW and by INFN research initiative PhenoLNF.

\section{References}

[1] D. Greynat and E. de Rafael, "Hadronic Contributions to the Muon Anomaly in the Constituent Chiral Quark Model", JHEP 1207, 020 (2012)

[2] G. Bennett et al., [Muon (g-2) Collaboration], Phys. Rev. D73 (2006) 072003.

[3] J.P. Miller, E. de Rafael and B.L. Roberts, Rep. Prog. Phys. 70 (2007) 795.

[4] F. Jegerlehner and A. Nyffeler, Phys. Rep. 477 (2009) 1.

[5] M. Davier, A. Hoecker, B. Malaescu and Z. Zhang, Eur. Phys. J. C71 (2011) 1515. 
[6] J. Prades, E. de Rafael and A. Vainshtein, Lepton Dipole Moments, Ch. 9 in Advanced Series on Directions in High Energy Physics-Vol. 20, Editors: B. Lee Roberts and William J. Marciano

[7] R. Carey et al, FERMILAB-PROPOSAL-0989,

[8] T. Mibe, Nucl. Phys. (Proc. Suppl.) B218 (2011) 242.

[9] A. Manohar and G. Georgi, Nucl. Phys. B233 (1984) 232.

[10] A. De Rújula, H. Georgi and S. Glashow, Phys.Rev. D12 (1975) 147

[11] S. Weinberg, Physica 96A (1984) 327.

[12] E. de Rafael, Phys. Lett. B703 (2011) 60.

[13] S. Weinberg, Phys.Rev.Lett. 105 (2010) 261601.

[14] C. Bouchiat and L. Michel, J. Phys. Radium 22 (1961) 121.

[15] S.J. Brodsky and E. de Rafael, Phys. Rev. 168 (1968) 1620.

[16] J. Calmet, S. Narison, M. Perrottet and E. de Rafael, Rev. Mod. Phys.49 (1977) 21.

[17] A.A. Pivovarov, Phys. Atom. Nucl. 66 (2003) 902.

[18] J. Erler and G.T. Sanchez, Phys. Rev. Lett. 97 (2006) 161801.

[19] G. Källen and A. Sabry, Kgl. Danske Videnskab. Selskab, Mat. Fys. Medd, 29 N. 17 (1955).

[20] B.E. Lautrup and E. de Rafael, Phys. Rev. 174 (1968) 1835.

[21] D. Espriu, E. de Rafael and J. Taron, Nucl. Phys. B 345 (1990) 22.

[22] $\mathrm{Xu}$ Feng et al (ETMC Collaboration), arXiv:1103.4818v1 [hep-lat].

[23] R. Barbieri and E. Remiddi, Nucl.Phys. B90 (1975) 273.

[24] K. Hagiwara et al, Phys. Rev. D69 (2004) 093003.

[25] B.E. Lautrup, A. Peterman and E. de Rafael, Phys. Reports 3C (1972) 193.

[26] E. de Rafael, Phys. Lett. B322 (1994) 239.

[27] M. Perrottet and E. de Rafael, unpublished.

[28] I. Blokland, A. Czarnecki and K. Melnikov, Phys. Rev. Lett. 88 (2002) 07183.
[29] N.N. Achasov et al., SND Collaboration, Eur. Phys. J. C12 (2000) 25; Phys. Lett. B559 (2003) 171.

[30] K. Hagiwara et al, J. Phys. G38 (2011) 085003.

[31] F. Jegerlehner, Nucl. Phys. Proc. Suppl. 162 (2006) 22.

[32] J. Aldins, S. Brodsky, A. Dufner and T. Kinoshita, Phys. Rev. Lett. 23 (1969) 441; Phys. Rev. D1 (1970) 2378.

[33] S. Laporta and E. Remiddi, , Phys. Lett., B301 (1993) 440 .

[34] R. Boughezal and K. Melnikov, Phys. Lett. B704 (2011) 114043

[35] M. Knecht and A. Nyffeler, Phys. Rev. D65 (2002) 073034.

[36] M. Knecht, A. Nyffeler, M. Perrottet and E. de Rafael, Phys. Rev. Lett. 88 (2002) 071802.

[37] J.-Ph. Aguilar, E. de Rafael and D. Greynat, Phys. Rev. D77 (2008) 093010.

[38] K. Melnikov and A. Vainshtein, Phys. Rev. D70 (2004) 113006.

[39] B. Babusci et al, arXiv:1109.2461v2 [hep-ph].

[40] J. Prades, E. de Rafael and A. Vainshtein, Lepton Dipole Moments, Ch.9, eds. B.L. Roberts and W.J. Marciano, World Scientific (2010).

[41] T. Goecke, C.S. Fisher and R. Williams, Prog. Part. Nucl. Phys. 67 (2012) 563.

[42] S. Peris, M. Perrottet and E. de Rafael, Phys. Lett. B355 (1995) 523.

[43] A. Czarnecki, B. Krause and W. Marciano, Phys. Rev. D52 (1995) R2619.

[44] M. Knecht, S. Peris, M. Perrottet and E. de Rafael, JHEP 0211 (2002) 003.

[45] A. Vainshtein, Phys. Lett. B569 (2003) 187.

[46] A. Czarnecki, W.J. Marciano and A. Vainshtein, Phys. Rev. D67 (2003) 073006 [Erratum-ibid. 73 (2006) 119901].

[47] M. Knecht, S. Peris, M. Perrottet and E. de Rafael, JHEP 0403 (2004) 035. 

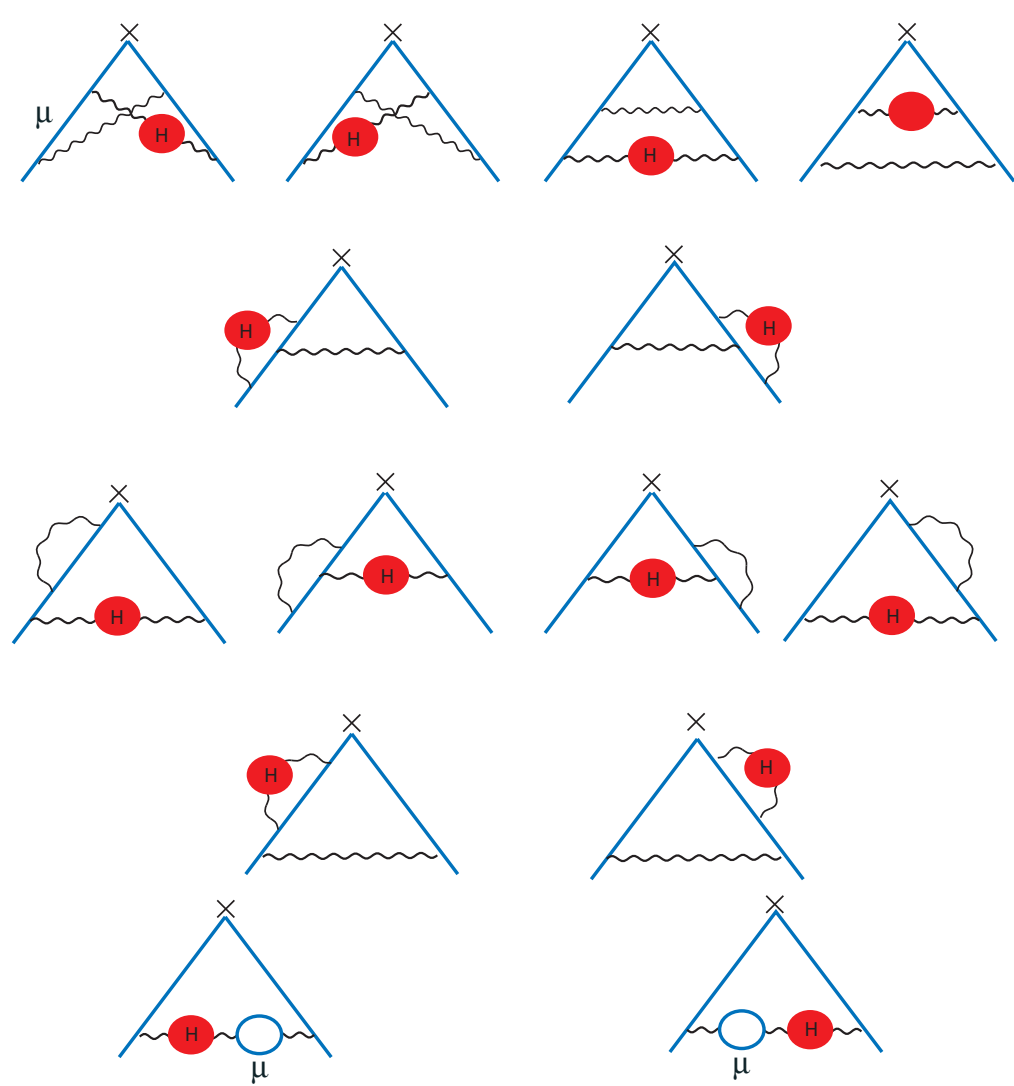

Figure 2. Feynman diagrams corresponding to the Class A contribution to the muon anomaly.
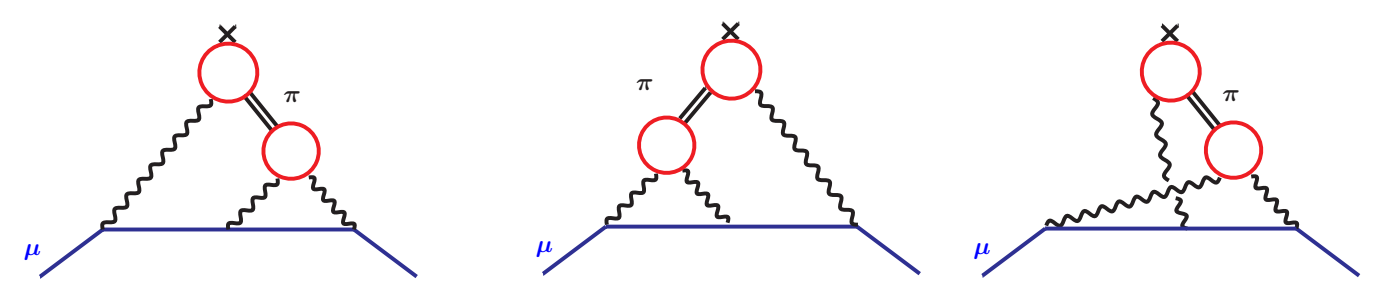

Figure 7. Goldstone Exchange Contribution to the Muon Anomaly in the $\mathrm{C} \chi \mathrm{QM}$.

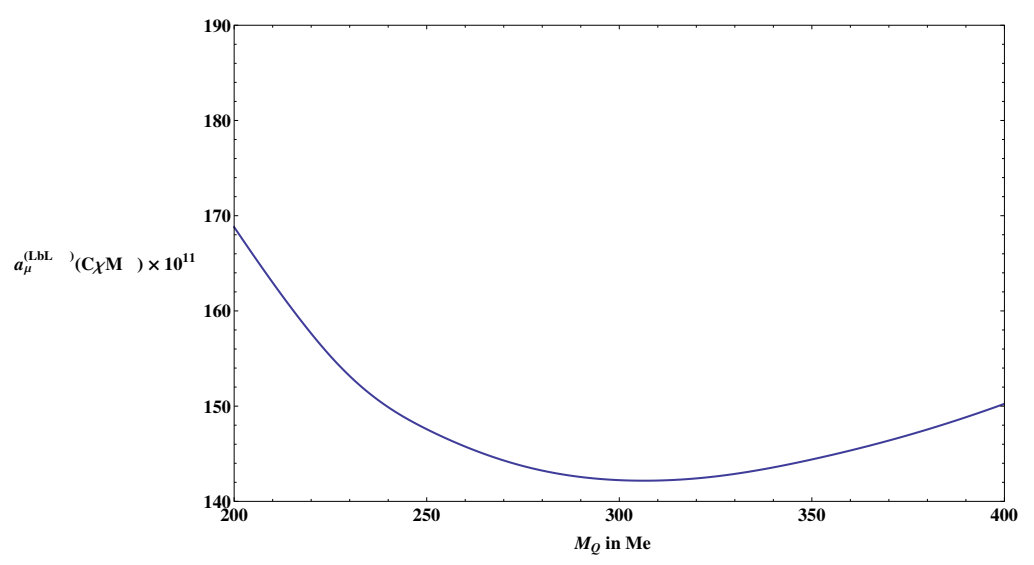

Figure 8. $a_{\mu}^{(\mathrm{HLbyL})}(\mathrm{C} \chi \mathrm{QM})$ versus $M_{Q}$ in $10^{-11}$ units. 